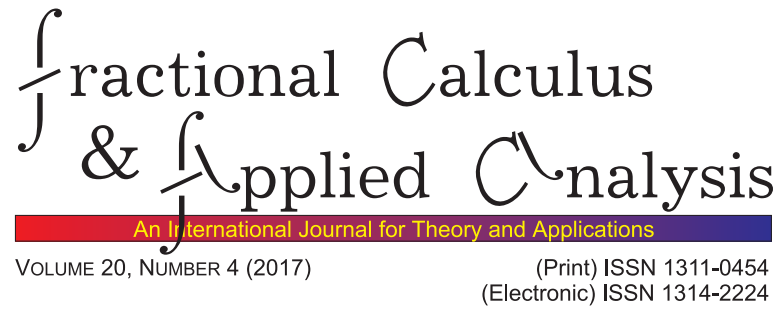

RESEARCH PAPER

\title{
ROBUSTNESS AND CONVERGENCE OF FRACTIONAL SYSTEMS AND THEIR APPLICATIONS TO ADAPTIVE SCHEMES
}

\author{
Javier A. Gallegos ${ }^{1}$, Manuel A. Duarte-Mermoud ${ }^{2}$
}

\begin{abstract}
Our general aim is to give sufficient conditions for robustness behavior and convergence to the equilibrium point of linear time-varying fractional system's solutions. We approach this problem using as a framework a series of recent results due to Cong et al. We establish theorems that generalize in several ways many previously published results, including those of Cong et al. We use the proposed theorems in control and adaptive systems, proving convergence and robustness of such schemes, that up to date remain as unsolved problems, showing the wide scope of applications of our results.
\end{abstract}

MSC 2010: Primary 26A33; Secondary 34A08, 34K37, 62F35

Key Words and Phrases: robustness, fractional differential equations, adaptive systems

\section{Introduction}

In recent papers [1, 2, 3, 4, Cong et al. have presented a fixed point technique to asymptotically analyze Caputo fractional systems with $\alpha \in$ $(0,1)$ described by the equation

$$
D^{\alpha} x(t)=(A+Q(t)) x(t)
$$

where we have implicitly assumed that the initial time for the derivative $t=0$, is the same that the time for the arbitrary initial condition $x_{0} \in \mathbb{R}^{n}$. They were able to prove ([1, Theorem 5]) that if $Q:[0, \infty) \rightarrow \mathbb{R}^{n \times n}$ is a continuous matrix function such that $\sup _{t \geq 0} \int_{0}^{t} \tau^{\alpha-1} \| E_{\alpha, \alpha}(A, \tau)[Q(t-$ $\tau)] \| d \tau<1$, where $E_{\alpha, \alpha}(A, t):=E_{\alpha, \alpha}\left(t^{\alpha} A\right)$ is the two-parameter Mittag-

(c) 2017 Diogenes Co., Sofia

pp. 895-913, DOI: $10.1515 /$ fca-2017-0047

DE GRUYTER 
Leffler function (see e.g. [6, Definition 4.2]) and if the spectrum $\sigma(A)$ of $A \in \mathbb{R}^{n \times n}$ is such that $\sigma(A) \subset\left\{\lambda \in \mathbb{C} \backslash\{0\}:|\arg (\lambda)|>\frac{\alpha \pi}{2}\right\}$, then $x$ is bounded, $\lim _{t \rightarrow \infty} x(t)=0$ and $x=0$ is Lyapunov stable. Actually, they show that instead of condition $q<1$ it is enough that $\sup _{t \geq 0}\|Q(t)\|$ be small enough or $\lim _{t \rightarrow \infty}\|Q(t)\|=0$ ([1, Theorems 7, 8]).

Under the same assumption for $A$ and similar arguments, for the system

$$
D^{\alpha} x(t)=A x(t)+f(x(t)),
$$

where $f(0)=0$ and $f$ is locally Lipschitz around the origin with $L(r)$ such that $\lim _{r \rightarrow 0^{+}} L(r)=0$ then $\lim _{t \rightarrow \infty}\|x(t)\|=0$ for any $\|x(0)\|<r_{0}$ and $r_{0}$ small enough [2, Theorem 5]. Roughly speaking, one can always find an $r$ such that $Q:=L(r) I$ is sufficiently small, and to apply the previous results. If $A$ is such that $\sigma(A) \subset\left\{\lambda \in \mathbb{C} \backslash\{0\}:|\arg (\lambda)|<\frac{\alpha \pi}{2}\right\}$, they prove that the origin $x=0$ is unstable ([3, Theorem 5]). The first Lyapunov Theorem on stability and instability follows as a corollary, i.e. for an autonomous system $D^{\alpha} x=f(x(t))$ with $\alpha \in(0,1)$ the local asymptotic stability of the origin is related to the eigenvalues of the Jacobian of $f$, a more general result that the one obtained using Lyapunov approach in [8, Example 4].

The alternative approach to study convergence and stability using Lyapunov functions $([8,[10])$ presents a disadvantage: by defining those functions as scalar ones, positive or negative definite restrictions on the matrix are required to establish the sign of its fractional derivatives ([8, Example $6])$.

Our contribution is first to extend the above theorems to include several new aspects such as: a wider notion of robustness, the use of time varying linear system, a piece-wise continuity requirement, the case of $\alpha \geq 1$ and other types of derivatives, and the extension to mixed order systems. Secondly, these extensions will be applied to robust control of fractional system and to robust convergence of adaptive systems without modifying the adaptive scheme but requiring conditions on the information signals, which ample the class of them that assure error convergence to zero when the perturbation is absent proposed in $([9])$.

The paper is organized as follows: Section 2 presents the main contribution of the paper, which is the generalization of the theorems just mentioned. Section 3 shows the usefulness of these generalizations by applying them to determine the convergence and robustness of adaptive scheme designs with fractional operators.

\section{Robustness and convergence}

We present theorems that extend in many aspects previous results in the revised literature. Though the arguments mainly follow the fixed point 
ROBUSTNESS AND CONVERGENCE OF FRACTIONAL ..

technique of Cong et al, fine details are required at each argumentation. The results have mathematical interest in itself and will show its importance in applications in Section 3 ,

We will use $\|\cdot\|_{\infty}$ to denote $L^{\infty}$ norm on functions, $\|\cdot\|$ for the standard Euclidean norm on $\mathbb{R}^{n}$ and also for any matrix norm compatible with this norm. We define the following sets which allow to classify stable and unstable matrices

$$
\begin{aligned}
& \Lambda_{\alpha}^{s}:=\left\{\lambda \in \mathbb{C} \backslash\{0\}:|\arg (\lambda)|>\frac{\alpha \pi}{2}\right\}, \\
& \Lambda_{\alpha}^{u}:=\left\{\lambda \in \mathbb{C} \backslash\{0\}:|\arg (\lambda)|<\frac{\alpha \pi}{2}\right\} .
\end{aligned}
$$

2.1. Robustness of linear time invariant fractional systems. Though [1, Theorem 5] is presented as a robustness result, because the convergence property is preserved when $Q(t)$ variations occur around $A$, a more general robustness condition can be stated where just the boundedness property of solutions is preserved. The following result considers this view and, at the same time, is a generalization to linear time varying systems of the sufficient part of [4, Theorem 13].

Theorem 2.1. Consider the following Caputo system

$$
D^{\alpha} x(t)=(A+Q(t)) x(t)+\nu(t)
$$

where $\alpha \in(0,1], x(t) \in \mathbb{R}^{n}$ for all $t \geq 0, \nu:[0, \infty) \rightarrow \mathbb{R}^{n}$ is a bounded continuous function and $A \in \mathbb{R}^{n \times n}$ is such that $\sigma(A) \subset \Lambda_{\alpha}^{s}$. Let $x_{0} \in \mathbb{R}^{n}$ be an arbitrary initial condition at the initial time of the Caputo derivative. Suppose that $Q:[0, \infty) \rightarrow \mathbb{R}^{n \times n}$ is a continuous matrix function such that

$$
q:=\sup _{t \geq 0}\left\|\int_{0}^{t} \tau^{\alpha-1} E_{\alpha, \alpha}(A, \tau) Q(t-\tau) d \tau\right\|<1
$$

Then the following statements hold:

(i) $x$ is a bounded continuous function.

(ii) If $\lim _{t \rightarrow \infty}\left\|\int_{0}^{t} \tau^{\alpha-1} E_{\alpha, \alpha}(A, \tau) \nu(t-\tau) d \tau\right\|=0$ then $\lim _{t \rightarrow \infty} x(t)=0$. In particular, if $\nu$ converges to zero, then $\lim _{t \rightarrow \infty} x(t)=0$ and if $\nu \equiv 0$ then $x=0$ is locally asymptotically stable.

P r o o f. Define the Lyapunov-Perron like operator as

$$
\begin{aligned}
\mathcal{T}_{x_{0}}(\xi)(t):= & E_{\alpha}\left(t^{\alpha} A\right) x_{0}+\int_{0}^{t} \tau^{\alpha-1} E_{\alpha, \alpha}(A, \tau) Q(t-\tau) \xi(t-\tau) d \tau \\
& +\int_{0}^{t} \tau^{\alpha-1} E_{\alpha, \alpha}(A, \tau) \nu(t-\tau) d \tau
\end{aligned}
$$


where $E_{\alpha}\left(t^{\alpha} A\right)$ is the Mittag-Leffler function (see e.g. [6, Definition 4.1]). Let $\epsilon>0$ arbitrary and $\xi \in B_{\infty}(0, \epsilon):=\left\{\xi \in \mathcal{C}\left([0, \infty) ; \mathbb{R}^{n}\right):\|\xi\|_{\infty} \leq\right.$ $\epsilon\}$. Using that $E_{\alpha}\left(t^{\alpha} A\right) x_{0}$ is bounded, $\int_{0}^{t} \tau^{\alpha-1}\left\|E_{\alpha, \alpha}\left(\tau^{\alpha} A\right)\right\| d \tau<\infty$ since $\sigma(A) \subset \Lambda_{\alpha}^{s}$ ([1, Theorem 3]), and that $\nu$ is bounded so that $\|\nu\|_{\infty}<\nu_{0}$, we have

$$
\left\|\mathcal{T}_{x_{0}}(\xi)\right\|_{\infty} \leq C\left(\epsilon, x_{0}, \nu_{0}\right)<\infty
$$

where $C\left(\epsilon,\left\|x_{0}\right\|, \nu_{0}\right)$ is a constant term depending on the bound $\nu_{0}$ of function $\nu$ and on $\epsilon,\left\|x_{0}\right\|$. Hence, $\mathcal{T}_{x_{0}}\left(B_{\infty}(0, \epsilon)\right) \subset B_{\infty}\left(0, C\left(\nu_{0}\right)\right)$ since the operator defines a continuous function from $[0, \infty) \rightarrow \mathbb{R}^{n}$ by the boundedness of its integrands. Taking $\tilde{\xi} \in \mathcal{C}([0, \infty)$, we have

$$
\left\|\mathcal{T}_{x_{0}}(\xi)-\mathcal{T}_{x_{0}}(\tilde{\xi})\right\|_{\infty} \leq q\|\xi-\tilde{\xi}\|_{\infty} .
$$

Therefore, $\mathcal{T}_{x_{0}}$ restricted to the Banach space $\mathcal{C}_{\infty}:=\left\{\xi \in \mathcal{C}\left([0, \infty) ; \mathbb{R}^{n}\right)\right.$ : $\left.\|\xi\|_{\infty}<\infty\right\}$ is a contraction auto map, whereby it has a unique fixed point $\xi_{0}$ by the contraction mapping theorem. Since the unique continuous solution to (2.3) can be written as $x(t)=E_{\alpha}\left(t^{\alpha} A\right) x_{0}+\int_{0}^{t} \tau^{\alpha-1} E_{\alpha, \alpha}(A, \tau) Q(t-$ $\tau) x(t-\tau) d \tau+\int_{0}^{t} \tau^{\alpha-1} E_{\alpha, \alpha}(A, \tau) \nu(t-\tau) d \tau=\mathcal{T}_{x_{0}}(x)(t)$ (see e.g [16, §3]), we conclude that $x=\xi_{0}$, and thus, $x$ is a bounded continuous function.

(ii) Since $x(t)=E_{\alpha}(A, t) x(0)+\int_{0}^{t} \tau^{\alpha-1} E_{\alpha, \alpha}(A, \tau) Q(t-\tau) x(t-\tau) d \tau$ $+\int_{0}^{t} \tau^{\alpha-1} E_{\alpha, \alpha}(A, \tau) \nu(t-\tau) d \tau$, we obtain

$$
\begin{aligned}
\|x(t)\| \leq & \left.\left\|E_{\alpha}(A, t) x(0)\right\|+\| \int_{0}^{t} \tau^{\alpha-1} E_{\alpha, \alpha}(A, \tau) Q(t-\tau) x(t-\tau)\right) d \tau \| \\
& +\left\|\int_{0}^{t} \tau^{\alpha-1} E_{\alpha, \alpha}(A, \tau) \nu(t-\tau) d \tau\right\| .
\end{aligned}
$$

Define $\epsilon:=\frac{1-q}{2 q+1} \lim \sup _{t \geq 0}\|x(t)\|$ and $T$ such that $\sup _{t \geq T}\|x(t)\| \leq$ $\lim \sup _{t \rightarrow \infty}\|x(t)\|+\epsilon$. By the part (i), $\epsilon, T$ exist since $x$ is bounded. Then,

$$
\limsup _{t \rightarrow \infty}\|x(t)\| \leq\left(\limsup _{t \rightarrow \infty}\|x(t)\|+\epsilon\right) \sup _{t \geq T}\left\|\int_{T}^{t} \tau^{\alpha-1} E_{\alpha, \alpha}(A, \tau) Q(t-\tau) d \tau\right\|
$$

where we use the hypothesis $\lim _{t \rightarrow \infty} \int_{0}^{t} \tau^{\alpha-1}\left\|E_{\alpha, \alpha}(A, \tau) \nu(t-\tau)\right\| d \tau=0$, the facts that $\left.\lim _{t \rightarrow \infty} \| \int_{0}^{T} \tau^{\alpha-1} E_{\alpha, \alpha}(A, \tau) Q(t-\tau) x(t-\tau)\right) d \tau \|$ and that since $\sigma(A) \subset \Lambda_{\alpha}^{s}, \lim _{t \rightarrow \infty}\left\|E_{\alpha}(A, t)\right\|=0$. Using condition (2.4), we obtain the following contradiction when $\lim \sup _{t \rightarrow \infty}\|x(t)\| \neq 0$,

$$
\limsup _{t \rightarrow \infty}\|x(t)\| \leq \limsup _{t \rightarrow \infty}\|x(t)\| \frac{2 q+q^{2}}{2 q+1}<\limsup _{t \rightarrow \infty}\|x(t)\| .
$$

Therefore, $\lim \sup _{t \rightarrow \infty}\|x(t)\|=0$ and $\lim _{t \rightarrow \infty} x(t)=0$.

Since $\sup _{t \geq 0} \int_{0}^{t} \tau^{\alpha-1}\left\|E_{\alpha, \alpha}(A, \tau)\right\| d \tau=C(\alpha, A)<\infty$ ([1, Theorem 3]), we have $\tau^{\alpha-1}\left\|E_{\alpha, \alpha}(A, \tau)\right\| \in \mathcal{L}^{1}$, the Lebesgue space of integrable functions 
on $\mathbb{R}_{\geq 0}$. If $\nu$ is bounded and converges to zero, we can apply [10, Lemma 2], to conclude that $\lim _{t \rightarrow \infty} \int_{0}^{t} \tau^{\alpha-1}|| E_{\alpha, \alpha}(A, \tau)|\||| \nu(t-\tau)\| d \tau=0$. Applying the previous part and taking limit on $0 \leq\left\|\int_{0}^{t} \tau^{\alpha-1} E_{\alpha, \alpha}(A, \tau) \nu(t-\tau) d \tau\right\| \leq$ $\int_{0}^{t} \tau^{\alpha-1}\left\|E_{\alpha, \alpha}(A, \tau) \mid\right\|\|\nu(t-\tau)\| d \tau$ we conclude $\lim _{t \rightarrow \infty} x(t)=0$. The local stability of $x=0$ when $\nu \equiv 0$ was proved in [1, Theorem 5].

REMARK 2.1. (i) When $\nu \equiv 0$ we recover [1, Theorem 5], and when $Q \equiv 0$ we recover the sufficiency part of [4, Theorem 13]. Moreover, the same arguments can be stated for complex valued solutions since $\mathcal{C}\left([0, \infty) ; \mathbb{C}^{n}\right)$ and its subset $\mathcal{C}_{\infty}$ are also Banach spaces.

(ii) From the form of the proof, we cannot get an estimate of the speed of convergence of $x$.

The following corollary generalizes [1, Theorem 6] and [1, Theorem 8] and shows, in its first claim, that hypothesis (2.4) is not trivial.

Corollary 2.1. Under the same hypotheses of Theorem 2.1 but using instead of condition (2.4), that there exists $T \geq 0$ such that $\sup _{t \geq T}\|Q(t)\|<$ $\frac{1}{C(\alpha, A)}$, where $C(\alpha, A):=\sup _{t \geq 0} \int_{0}^{t} \tau^{\alpha-1}\left\|E_{\alpha, \alpha}(A, \tau)\right\| d \tau<\infty$, claims (i) and (ii) hold. In particular, if $\lim _{t \rightarrow \infty} Q(t)=0$ claims (i) and (ii) hold.

P r o o f. By the condition on $\sigma(A), \sup _{t>0}\left\|\int_{0}^{t} \tau^{\alpha-1} E_{\alpha, \alpha}(A, \tau) d \tau\right\|=$ $C(\alpha, A)<\infty\left(\left[1\right.\right.$, Theorem 3]). If $\sup _{t \geq 0}\|Q(t)\|<\frac{1}{C(\alpha, A)}$, we conclude that condition (2.4) holds.

On the other hand, if $\lim _{t \rightarrow \infty}\|Q(t)\|=0$, then there exists $T$ such that $\sup _{t>T}\left\|\int_{0}^{t} \tau^{\alpha-1} E_{\alpha, \alpha}(A, \tau)[Q(t-\tau)]\right\|<1$. Following the reasoning of the proof of Theorem 2.1, we have for $\xi \in \mathcal{C}_{\infty}$

$$
\begin{gathered}
\xi(t)=E_{\alpha}(A, t) x(0)+\int_{0}^{T}(t-\tau)^{\alpha-1} E_{\alpha, \alpha}(A, t-\tau) Q(\tau) \xi(\tau) d \tau \\
+\int_{T}^{t} \tau^{\alpha-1} E_{\alpha, \alpha}(A, \tau) Q(t-\tau) \xi(t-\tau) d \tau+\int_{0}^{t} \tau^{\alpha-1} E_{\alpha, \alpha}(A, \tau) \nu(t-\tau) d \tau
\end{gathered}
$$

The first integral of right-hand side converges to zero by applying [11, Property 15] since $\left\|\int_{0}^{T}(t-\tau)^{\alpha-1} E_{\alpha, \alpha}(A, t-\tau) Q(\tau) \xi(\tau) d \tau\right\| \leq C_{E} \int_{0}^{T}(t-$ $\tau)^{\alpha-1}\|Q(\tau) \xi(\tau)\| d \tau$. The rest follows from similar arguments of the proof of Theorem 2.1.

REMARK 2.2. Note, for control applications, that condition (2.4) or $\sup _{t}\|Q(t)\|<\frac{1}{C(\alpha, A)}$ depends on $A$ (more specifically on the magnitude of 
its eigenvalues) and $\alpha$. By choosing $A, \alpha$ we expand the range of matrices $Q$ assuring convergence (at least in principle, since the result is just a sufficient statement).

2.2. Comparison property. The following result generalizes [1, Theorem 5 ] in the sense that matrix $A$ can now be a time varying function and the condition on $A(\cdot)$ is given as an inequality rather than an equality of type $A+Q(t)$. It can be seen as a comparison result generalizing that of [10, Theorem 4].

Theorem 2.2. Consider the following Caputo system

$$
D^{\alpha} x(t)=A(t) x(t)+\nu(t),
$$

with $\alpha \in(0,1), x(t) \in \mathbb{R}^{n}$ for all $t \geq 0$ and $\nu:[0, \infty) \rightarrow \mathbb{R}^{n}$ a bounded differentiable function. Suppose that $A:[0, \infty) \rightarrow \mathbb{R}^{n \times n}$ is a differentiable matrix function satisfying $A(t) \leq-\epsilon I+Q(t)$ where $I$ is the identity matrix, $Q:[0, \infty) \rightarrow \mathbb{R}^{n \times n}$ is a bounded continuous matrix function and $\epsilon>0$. Then,

(i) If $\sup _{t}\|Q(t)\|<\mu<\epsilon$, then the solution $x$ is a bounded continuous function.

(ii) If $q=\sup _{t \geq 0}\left|\int_{0}^{t} \tau^{\alpha-1} E_{\alpha, \alpha}\left(-\epsilon t^{\alpha}\right)\left[\lambda_{M}(t-\tau)\right]\right| d \tau<1$, where $\lambda_{M}(t)$ is the largest eigenvalue of $Q(t)$ and $\nu \equiv 0$, then $\lim _{t \rightarrow \infty} x(t)=0$.

P r o o f. (i) We have

$$
\begin{array}{r}
1 / 2 D^{\alpha}\left[x^{T} x\right] \leq x^{T} D^{\alpha} x=x^{T} A x+x^{T} \nu \\
\leq-\epsilon x^{T} x+x^{T} Q x+x^{T} \nu \leq-\epsilon x^{T} x+\mu x^{T} x+x^{T} \nu,
\end{array}
$$

where the first inequality is due to [7, Lemma 1] which can be applied since $\nu$ and $A$ are bounded continuous functions, whereby $x$ is a differentiable function [10, Property 12].

Given that $Q$ and $\nu$ are bounded functions, the set $\Omega=\left\{x \in \mathbb{R}^{n} \mid \epsilon x^{T} x-\right.$ $\left.\mu x^{T} x-x^{T} \nu \leq 0\right\}$ is bounded (otherwise the positive term $(\epsilon-\mu) x^{T} x$ would dominate leading to a contradiction). Whereby $\bar{\Omega}$ is compact and therefore $x$ is bounded by [10, Theorem 8].

(ii) As in part (i), we have

$$
1 / 2 D^{\alpha}\left[x^{T} x\right] \leq x^{T}(-\epsilon I+Q) x \leq-\epsilon x^{T} x+\lambda_{M}(t) x^{T} x .
$$

By using Theorem 2.1 on the equation $1 / 2 D^{\alpha} y=-\epsilon y+\lambda_{M}(t) y$, we conclude that $\lim _{t \rightarrow \infty} y(t)=0$, and hence by comparison [10, Lemma 1], $\lim _{t \rightarrow \infty} x(t)=0$.

REMARK 2.3. If $\|Q\|$ converges to zero, then $\lambda_{M}$ also does it and applying Corollary 2.1, we have $\lim _{t \rightarrow \infty} x=0$. Similarly if $\sup _{t}\|Q(t)\|<$ 
ROBUSTNESS AND CONVERGENCE OF FRACTIONAL ... 901

$\frac{1}{C(\alpha, A)}$ for an induced matrix norm (for it, the bound of the matrix norm bounds its largest eigenvalue).

2.3. Extensions for $\alpha \geq 1$ and other types of derivatives. The starting point of Cong et al. is the analytic-implicit solution of equation (1.1). For $1<\alpha \leq 2$, it takes the form [12, Theorem 5.15]

$x(t)=E_{\alpha}\left(t^{\alpha} A\right) x_{0}+t E_{\alpha, 2}\left(t^{\alpha} A\right) \dot{x}_{0}+\int_{0}^{t} \tau^{\alpha-1} E_{\alpha, \alpha}(A, \tau) Q(t-\tau) x(t-\tau) d \tau$,

where $x_{0}, \dot{x}_{0} \in \mathbb{R}^{n}$ are initial conditions associated to the function $x$ and its derivative, respectively.

This can be directly proved by applying the Laplace transform. Moreover, it can be generalized for any order of derivation by the same procedure. However, for $\alpha \geq 2$, there is no condition for a stable constant matrix, since $\left\{\lambda \in \mathbb{C} \backslash\{0\}:|\arg (\lambda)|>\frac{\alpha \pi}{2}\right\}=\varnothing$ for $\alpha \geq 2$.

In addition, one can verify uniqueness of the solution (see for instance [6. Theorem 6.5], [12, Theorem 3.25] and the global version in [17]) provided that $Q$ is a bounded function. Now we can formulate the following extension to [1, Theorem 5], which is a generalization of [10, Theorem 4].

Theorem 2.3. Consider the following Caputo system

$$
D^{\alpha} x(t)=(A+Q(t)) x(t)+\nu(t)
$$

with $0<\alpha<2, x:[0, \infty) \rightarrow \mathbb{R}^{n}$ and $\nu:[0, \infty) \rightarrow \mathbb{R}^{n}$ a bounded continuous function. Let $x_{0}, \dot{x}_{0} \in \mathbb{R}^{n}$ be arbitrary initial conditions. Suppose that $Q:[0, \infty) \rightarrow \mathbb{R}^{n \times n}$ is a continuous matrix function such that $q=\sup _{t \geq 0}\left\|\int_{0}^{t} \tau^{\alpha-1} E_{\alpha, \alpha}(A, \tau)[Q(t-\tau)] d \tau\right\|<1$. Assume that $A \in \mathbb{R}^{n \times n}$ is such that $\sigma(A) \subset \Lambda_{\alpha}^{s}$. Then,

(i) $x$ is bounded continuous function.

(ii) If in addition $\lim _{t \rightarrow \infty}\left\|\int_{0}^{t} \tau^{\alpha-1} E_{\alpha, \alpha}(A, \tau) \nu(t-\tau) d \tau\right\|=0$ then $\lim _{t \rightarrow \infty}\|x(t)\|=0$. In particular, if $\nu$ converges to zero, then $\lim _{t \rightarrow \infty} x(t)=$ 0 and if $\nu \equiv 0$ then the point $(x, \dot{x})=(0,0)$ is asymptotically stable.

P r o o f. The proof is similar to the proof of Theorem 2.1. We just indicate the points essentially different. By applying Laplace Transform we conclude that the solution to (2.7) must satisfy the expression (2.6). The Lyapunov-Perron operator is then defined by

$$
\begin{aligned}
\mathcal{T}_{x_{0}, \dot{x}_{0}}(\xi)(t):= & E_{\alpha}\left(t^{\alpha} A\right) x_{0}+t E_{\alpha, 2}\left(t^{\alpha} A\right) \dot{x}_{0} \\
& +\int_{0}^{t} \tau^{\alpha-1} E_{\alpha, \alpha}(A, \tau)[Q(t-\tau) \xi(t-\tau)+\nu(t-\tau)] d \tau
\end{aligned}
$$


By taking $\xi, \tilde{\xi} \in B_{\infty}(0, \epsilon)$, we have $\left\|\mathcal{T}_{x_{0}, \dot{x}_{0}}(\xi)-\mathcal{T}_{x_{0}, \dot{x}_{0}}(\tilde{\xi})\right\|_{\infty} \leq q\|\xi-\tilde{\xi}\|_{\infty}$. Since $\sigma(A) \subset \Lambda_{\alpha}^{s}$ the initial conditions terms are bounded, decaying to zero ([18, Lemma 3(i)]) and $\left\|\int_{0}^{t} \tau^{\alpha-1} E_{\alpha, \alpha}(A, \tau) d \tau\right\|<\infty$ ([1, Theorem 3]). Since $\nu$ is bounded, $\left\|\mathcal{T}_{x_{0}, \dot{x}_{0}}(\xi)\right\|_{\infty}<\infty$.

Since $q<1, \mathcal{T}_{x_{0}, \dot{x}_{0}}$ restricted to $\mathcal{C}_{\infty}$ is a contraction auto map in a Banach space, whereby it has a unique fixed point. Since the unique continuous solution [6] to $D^{\alpha} x=(A+Q(t)) x$ can be written as $x(t)=$ $E_{\alpha}\left(t^{\alpha} A\right) x_{0}+t E_{\alpha, 2}\left(t^{\alpha} A\right) \dot{x}_{0}+\int_{0}^{t} \tau^{\alpha-1} E_{\alpha, \alpha}(A, \tau)[Q(t-\tau) \xi(t-\tau)+\nu(t-\tau)] d \tau$ we conclude that $x=\xi$, whereby $x$ is a bounded continuous function and $\lim \sup _{t \rightarrow \infty}\|x(t)\|<\infty$.

Theorem 2.3 can be generalized to other type of fractional derivatives provided that, by applying the Laplace transform, one has (i) $\mathcal{L}\left[D^{\alpha} f\right](s)=$ $s^{\alpha} f(s)-F(0, s)$ for any continuous function $f$ where $F(0, s)$ is an initial condition term associated to the specific fractional derivative (hence, the implicit solution to (2.7) can be written in Laplace domain as $x(s)=\left(s^{\alpha}-\right.$ $\left.I)^{-1} F(0, s)+\left(s^{\alpha}-I\right)^{-1}[Q x](s)\right)$, (ii) uniqueness of the continuous solution for system (2.7) and (iii) the term $\mathcal{L}^{-1}[F(0, s)](t)$ converges to zero provided that $\sigma(A) \subset \Lambda_{\alpha}^{s}$ and is bounded from some $T>0$. For instance, RiemannLiouville derivative satisfies the above conditions under mild assumptions (see [16, §2.8] for (i), [12, §3] for (ii), and [2, Proposition 4] and null initial conditions for (iii)).

We now extend the first Lyapunov theorem in its stability-instability part for $\alpha>1$. It will appear as a direct consequence of a more general result. When $\alpha<1$, the stability was proved in [2, Theorem 5] and the instability in [3, Theorem 8]. This theorem also is more general that the result of [18, Theorem 4], since it considers the unstable case.

Though the function $h(x)=A x+f(x)$ (where $h(0)=0$ ) does not depend on $\dot{x}$, for $\alpha>1$ the equilibrium point must consider this variable; it cannot be calculated by just asking for those $x$ such that $h(x)=0$. By uniqueness and closed inspection of equation (2.6) with $Q x$ replaced by $f(x)$, we conclude that if $\left(x_{0}, \dot{x}_{0}\right)=(0,0)$ then the consistent solution is $x \equiv 0$, thus $\left(x^{T}, \dot{x}^{T}\right)^{T}=(0,0)^{T}$ is an equilibrium point.

Theorem 2.4. Consider the following Caputo system for $0<\alpha<2$

$$
D^{\alpha} x=A x+f(x),
$$

with $x:[0, \infty) \rightarrow \mathbb{R}^{n}$ and $f:[0, \infty) \rightarrow \mathbb{R}^{n}$ a locally Lipschitz function with Lipschitz constant such that $\lim _{r \rightarrow 0^{+}} L(r)=0$ and $f(0)=0$.

(i) If $\sigma(A) \subset \Lambda_{\alpha}^{s}$ then $(x, \dot{x})=(0,0)$ is a locally asymptotically stable equilibrium point.

(ii) If $A$ has at least one eigenvalue in $\Lambda_{\alpha}^{u}$ then $(x, \dot{x})=(0,0)$ is an unstable equilibrium point. 
$\mathrm{P}$ r o o f. (i) The proof is similar to that of Theorem 2.3, provided that $\sup _{t>0}\left\|\int_{0}^{t} \tau^{\alpha-1} E_{\alpha, \alpha}(A, \tau)\right\| d \tau<\infty$ for $\alpha>1$ and $\sigma(A) \subset \Lambda_{\alpha}^{s}$.

In the scalar case $\int\left|\tau^{\alpha-1} E_{\alpha, \alpha}(A, \tau)\right| d \tau<\infty$ is equivalent to determine if $\left|\tau^{\alpha-1} E_{\alpha, \alpha}(A, \tau)\right| \in \mathcal{L}^{1}$ which was asserted in [13, Theorem 2].

Since $\tau^{\alpha-1} E_{\alpha, \alpha}(A, \tau)=\mathcal{L}^{-1}\left[\left(s^{\alpha} I-A\right)^{-1}\right]$ and $\sigma(A) \subset \Lambda_{\alpha}^{s}$, the transfer function $h(t)=c^{T} \mathcal{L}^{-1}\left[\left(s^{\alpha} I-A\right)^{-1}\right](t) b$ by [13, Theorem 2] $h(t) \in \mathcal{L}^{1}$ since $\hat{h}=\mathcal{L}[h](s)$ has positive relative degree. In particular, choosing $c, b$ as $e_{i}, e_{j}$ elements of the canonical base of $\mathbb{R}^{n}$, it follows that every element of matrix $\mathcal{L}^{-1}\left[\left(s^{\alpha} I-A\right)^{-1}\right]$ belongs to $\mathcal{L}^{1}$. Since $\|A\|_{1}=\sup _{i, j}\left|a_{i j}\right|<\sum_{i, j=1}^{n}\left|a_{i j}\right|$, we have $\left\|\tau^{\alpha-1} E_{\alpha, \alpha}(A, \tau)\right\|_{1} \in \mathcal{L}^{1}$ (the chosen matrix norm is equivalent to others norms such as the Frobenius norm and $\|\cdot\|_{2}$, whereby it is compatible with the standard norm on $\left.\mathbb{R}^{n}\right)$.

Since $\|f(x)\| \leq L(r)\|x\|$, we can choose a sufficiently small $r$ so that $\sup _{t \geq 0} \int_{0}^{t} \tau^{\alpha-1}\left\|E_{\alpha, \alpha}(A, \tau) L(r) \xi(t-\tau)\right\| d \tau<1$ for $\xi \in B_{\infty}(0, r)$. The rest is similar to the proof of Theorem 2.3.

(ii) Suppose that $x=0, \dot{x}=0$ is an stable point. Then for all $\epsilon>0$ there exists $\delta$ such that if $\left(x_{0}^{T}, \dot{x}_{0}^{T}\right)^{T} \in B_{\mathbb{R}^{n}}(0, \delta)$ then $\left(x^{T}, \dot{x}^{T}\right)^{T} \in B_{\mathbb{R}^{n}}(0, \epsilon)$. In particular, if $\left(x_{0}^{T}, 0^{T}\right)^{T} \in B_{\mathbb{R}^{n}}(0, \delta)$ then $x \in B_{\mathbb{R}^{n}}(0, \epsilon)$. By a Jordan canonical transformation and without loss of generality, we can suppose $A$ a diagonal matrix of eigenvalues with its first simple eigenvalue $\lambda_{1}$ belonging to $\Lambda_{\alpha}^{u}$ and all the rest in $\Lambda_{\alpha}^{s}$. Therefore, the solution to (2.8) with initial condition $\left(x(0)^{T}, \dot{x}(0)^{T}\right)=\left(x_{0}^{1}, 0, \ldots, 0\right)$ can be expressed for its first component $x^{1}$ as

$$
x^{1}(t)=E_{\alpha}\left(\lambda_{1} t^{\alpha}\right) x_{0}^{1}+\int_{0}^{t} \tau^{\alpha-1} E_{\alpha, \alpha}(A, \tau) f^{1}(x(t-\tau)) d \tau .
$$

By [3, Lemma 4] - a result directly deduced from [16, Theorems 1.3, 1.4] which has validity for $0<\alpha<2$ - we have

$$
\lim _{t \rightarrow \infty} \int_{0}^{t} \frac{\tau^{\alpha-1} E_{\alpha, \alpha}\left(\lambda \tau^{\alpha}\right) f^{1}(x(t-\tau))}{E_{\alpha}\left(\lambda_{1} t^{\alpha}\right)} d \tau=\lambda_{1}^{\frac{1-\alpha}{\alpha}} \int_{0}^{t} \exp \left(-\lambda_{1}^{\frac{1-\alpha}{\alpha}} \tau\right) f^{1}(t-\tau) d \tau .
$$

Since $E_{\alpha}\left(\lambda_{1} t^{\alpha}\right)$ diverges and $\left\|x^{1}\right\|_{\infty}<\epsilon$ by our assumption of stability, necessarily

$$
x_{0}^{1}=-\lambda_{1}^{\frac{1-\alpha}{\alpha}} \int_{0}^{t} \exp \left(-\lambda_{1}^{\frac{1-\alpha}{\alpha}} \tau\right) f^{1}(x(t-\tau)) d \tau .
$$

Defining

$$
\begin{aligned}
\mathcal{T} \xi^{1}:= & \int_{0}^{t} \tau^{\alpha-1} E_{\alpha, \alpha}\left(\lambda \tau^{\alpha}\right) f^{1}(\xi(t-\tau)) d \tau \\
& -\lambda_{1}^{\frac{1-\alpha}{\alpha}} E_{\alpha}\left(\lambda_{i} t^{\alpha}\right) \int_{0}^{t} \exp \left(-\lambda_{1}^{\frac{1-\alpha}{\alpha}} \tau\right) f^{1}(\xi(t-\tau)) d \tau
\end{aligned}
$$




$$
\mathcal{T} \xi^{i}:=\int_{0}^{t} \tau^{\alpha-1} E_{\alpha, \alpha}\left(\lambda \tau^{\alpha}\right) f^{i}(\xi(t-\tau)) d \tau,
$$

for $i=2, \ldots, n$, we observe that the solution to (2.8) with initial condition $\left(x(0)^{T}, \dot{x}(0)^{T}\right)=\left(x_{0}^{1}, 0, \ldots, 0\right)^{T}$ must be a fixed point of $\mathcal{T}$.

Let $\xi \in B_{\infty}(0, \epsilon)$. Using, [3, Lemma 3], which has validity for $0<$ $\alpha<2$, we have $\left\|\mathcal{T} \xi^{1}\right\|_{\infty} \leq K\left(\alpha, \lambda_{1}\right)\|f\|_{\infty}$ and using part (i), we have for $i=2, \ldots, n\left\|\mathcal{T} \xi^{i}\right\|_{\infty} \leq C(\alpha, A)\|f\|_{\infty}$. Choosing $\epsilon$ such that $\left(K\left(\alpha, \lambda_{1}\right)+\right.$ $C(\alpha, A)) l(\epsilon)<1$, we have

$$
\|x\|_{\infty}=\|\mathcal{T} x\|_{\infty} \leq C(\alpha, A) l(\epsilon)\|x\|_{\infty}<\|x\|_{\infty}
$$

which is a contradiction for $x_{0}^{1} \neq 0$. Then $\left(x^{T}, \dot{x}^{T}\right)^{T}=(0,0)^{T}$ is an unstable point.

REMARK 2.4. From a Taylor expansion around the origin, namely $f(x)=J_{f}(0) x+p(x(t))$ where, $f(0)=0, p$ is locally Lipschitz around the origin with Lipschitz constant such that $\lim _{r \rightarrow 0^{+}} L(r)=0$ since it is a polynomial on $x$ and $J_{f}(x=0)=J_{f}(0)$ the Jacobian of $f$ at zero, we have (First Lyapunov Theorem): if $\sigma\left(J_{f}(0)\right) \subset \Lambda_{\alpha}^{s}$ then local asymptotic stability of the origin $(x, \dot{x})=(0,0)$ follows and instability if at least one eigenvalue belongs to $\Lambda_{\alpha}^{u}$. We recall that even for integer order systems, the stability cannot be asserted from this local analysis if there exist eigenvalues such that $\left\{\lambda \in \mathbb{C} \backslash\{0\}:|\arg (\lambda)|=\frac{\alpha \pi}{2}\right\}$. Note also that instability does not necessarily imply that the solution diverges as in [4, Proposition 6], where is proved that there exists initial conditions (holding equation (2.9)) such that the solution remains bounded for unstable systems.

We will consider the autonomous systems $D^{\alpha} x=f(x)$ perturbed around the equilibrium point $x=0$ in its Taylor expansion. We use the concept of locally ultimately bound equilibrium point meaning that the solutions starting near enough of the equilibrium point remain bounded.

Corollary 2.2. Consider the Caputo system

$$
D^{\alpha} x(t)=(A+Q(t)) x(t)+f(x(t))+\nu(t),
$$

where $0<\alpha<2, x(t) \in \mathbb{R}^{n}$ for all $t \geq 0, \nu:[0, \infty) \rightarrow \mathbb{R}^{n}$ a bounded Lipschitz continuous function, $A$ and $f$ as in Theorem 2.4 and $Q:[0, \infty) \rightarrow$ $\mathbb{R}^{n \times n}$ as in Theorem 2.3 (or Corollary 2.1). Then,

(i) $x=0, \dot{x}=0$ is a locally ultimately bound equilibrium point.

(ii) If in addition $\lim _{t \rightarrow \infty}\left\|\int_{0}^{t} \tau^{\alpha-1} E_{\alpha, \alpha}(A, \tau) \nu(t-\tau) d \tau\right\|=0$ then $\lim _{t \rightarrow \infty}\|x(t)\|=0$. In particular, if $\nu$ converges to zero, then $x=0, \dot{x}=0$ is an attractive equilibrium point and if $\nu \equiv 0$ it is an asymptotically stable point. 
ROBUSTNESS AND CONVERGENCE OF FRACTIONAL ... 905

P r o o f. (i) We present the proof for $0<\alpha<1$ since for $1 \leq \alpha<2$ the analysis is similar. The Lyapunov-Perron operator is defined as

$$
\begin{aligned}
\mathcal{T}_{x_{0}}(\xi)(t):= & E_{\alpha}\left(t^{\alpha} A\right) x_{0}+\int_{0}^{t} \tau^{\alpha-1} E_{\alpha, \alpha}(A, \tau) Q(t-\tau) \xi(t-\tau) d \tau \\
& +\int_{0}^{t} \tau^{\alpha-1}\left[E_{\alpha, \alpha}(A, \tau) f(\xi(t-\tau))+E_{\alpha, \alpha}(A, \tau) \nu(t-\tau)\right] d \tau .
\end{aligned}
$$

Let $\epsilon>0$. By taking $\xi, \hat{\xi}(t) \in B_{\infty}(0, \epsilon)$ and using that $E_{\alpha}\left(t^{\alpha} A\right) x_{0}$ is bounded, $\int_{0}^{t} \tau^{\alpha-1}\left\|E_{\alpha, \alpha}\left(\tau^{\alpha} A\right)\right\| d \tau=C(\alpha, A)<\infty$ (since $\left.\sigma(A) \subset \Lambda_{\alpha}^{s}\right)$ and since by Lipschitz assumption, $\|f(\xi)-f(\hat{\xi})\|_{\infty} \leq L(\epsilon)\|\xi-\hat{\xi}\|_{\infty}$, we have

$$
\left\|\mathcal{T}_{x_{0}} \xi-\mathcal{T}_{x_{0}} \hat{\xi}\right\|_{\infty} \leq q\|\xi-\hat{\xi}\|_{\infty}+C(\alpha, A) L(\epsilon)\|\xi-\hat{\xi}\|_{\infty}
$$

By taking $\hat{\xi}=0$, we get $\left\|\mathcal{T}_{x_{0}}(\xi)\right\|_{\infty} \leq C\left(\nu_{0}\right)+q \epsilon+C(\alpha, A) L(\epsilon) \epsilon$. Thus, $\mathcal{T}_{x_{0}}\left(B_{\infty}(0, \epsilon)\right) \subset B_{\infty}\left(0, C\left(\nu_{0}\right)\right)$. Since $\lim _{r \rightarrow 0^{+}} L(r)=0$, we can choose a sufficiently small $\epsilon<1$ so that $\mu=q+C(\alpha, A) L(\epsilon)<1$ and $\mathcal{T}_{x_{0}}$ is a contraction auto map, whereby it has a unique fixed point $\xi_{0}$. Since the unique solution to $D^{\alpha} x=F(t, x):=(A+Q(t)) x+f(x(t))+\nu(t)$ (uniqueness follows from Lipschitz continuity of $F$ in the second variable) can be written as $x(t)=E_{\alpha}\left(t^{\alpha} A\right) x_{0}+\int_{0}^{t} \tau^{\alpha-1} E_{\alpha, \alpha}(A, \tau)[Q(t-\tau) \xi(t-\tau)+f(x(t-\tau))+$ $\nu(t-\tau)] d \tau$, we conclude that $x=\xi_{0}$ when $x_{0} \in B_{\mathbb{R}^{n}}(0, \epsilon)$, whereby $x$ is a bounded continuous function.

(ii) It is similar to the proof of Theorem 2.1(ii) and hence omitted.

We state the following linearization result, which, based in reference [2, Section 1] where the literature is carefully revised, have been applied without a rigorous proof.

Corollary 2.3. Consider the following Caputo system

$$
D^{\alpha} x=f(x, t)
$$

where $0<\alpha<2, f(x, t)$ an smooth function such that $f(0, \cdot) \equiv 0$ and $\lim _{t \rightarrow \infty} f(x, t)=g(x)$ with $g(0)=0$ and the Jacobian of $g$ satisfying $\sigma\left(J_{g}(0)\right) \subset \Lambda_{\alpha}^{s}$. Then $x=0$ is locally asymptotically stable.

P r o o f. Using that $\lim _{t \rightarrow \infty} f(x, t)=g(x)$ and that $f(0, \cdot) \equiv 0$, the local expansion around $x=0$ of $f$ must hold that $f(x, t)=\left(J_{g}(0)+\right.$ $Q(t)) x+q(x(t))+\nu(x(t))$ where $Q, \nu$ vanish asymptotically and $q$ is such that $g(x)=J_{g}(0) x+q(x)$ for a sufficiently small $x$. The rest follows from applying Corollary 2.2(ii)

As an example of function holding Corollary 2.3 consider $f(x, t)=$ $g(x)+u(t) h(x)$ where $u(t) \rightarrow 0$ as $t \rightarrow \infty$, and $\sigma\left(J_{g}(0)\right) \subset \Lambda_{\alpha}^{s}$. 
Example 2.1. Local robust control of non linear system. Consider the system

$$
D^{\alpha} x=f(x, u)
$$

where $0<\alpha<2, f$ is a smooth function with $f(0, \cdot)=0$ and $u=K x$ with $K$ constant matrix to be determined. By Taylor expansion around $x=0$, $f(x, u)=J_{x}(0,0) x+J_{u}(0,0) u+p(x)$, where $J$ is the Jacobian of $f$ and $p$ is a polynomial. If the pair $\left(J_{f}(0,0), J_{u}(0,0)\right)$ is controllable, there exists $K_{A}$ such that for any $A, J_{f}(0,0)+J_{u}(0,0) K_{A}=A$. Choosing $A$ such that $\sigma(A) \subset \Lambda_{\alpha}^{s}$, then Corollary 2.2 implies that the control $u=K_{A} x$ is robust for any initial condition sufficiently close to $x=0$.

2.4. Extensions for piece-wise continuous functions. The function $Q(t)$ had been required to be a continuous function. We extend the results for the case when $Q(t)$ is only piece-wise continuous, namely there exist intervals $\left[T_{i}, T_{i+1}\right)$ for $i \in \mathbb{N}$ such that $Q(t)$ is continuous in $\left[T_{i}, T_{i+1}\right)$. This problem is relevant in switching systems; for instance $Q(t)$ could be a constant matrix in $\left[T_{i}, T_{i+1}\right)$.

Theorem 2.5. Consider the Caputo system of (2.7) with the same requirement of Theorem 2.3 but now $\nu, Q$ are allowed to be piece-wise continuous function. Then, $x$ is a bounded continuous function. If in addition $\lim _{t \rightarrow \infty}\left\|\int_{0}^{t} \tau^{\alpha-1} E_{\alpha, \alpha}(A, \tau) \nu(t-\tau) d \tau\right\|=0$, then $\lim _{t \rightarrow \infty}\|x(t)\|=$ 0 . In particular, if $\nu$ converges to zero, then $\lim _{t \rightarrow \infty}\|x(t)\|=0$ and if $\nu \equiv 0$ then the point $(x, \dot{x})=(0,0)$ is asymptotically stable.

P r o o f. The proof is similar that of Theorem 2.3 if the solution to (2.7), with piece-wise continuous functions $\nu, Q$, (i) can be expressed in terms of $E_{\alpha}$ functions and (ii) is the unique continuous function satisfying the given initial condition.

Part (i) follows by applying Laplace transform, since it also applies on piece-wise continuous functions. The resultant expression is the same as (2.6) plus the extra term $\int_{0}^{t}(t-\tau)^{\alpha-1} E_{\alpha, \alpha}(A, t-\tau) \nu(\tau) d \tau$ due to the perturbation.

For (ii), let $\nu \equiv 0$. If $x_{0}=0, \dot{x}_{0}=0$ then there is a unique solution $x \equiv 0$ in the first interval $\left[T_{0}, T_{1}\right)$ since it is indistinguishable of the continuous case $([12$, Theorem 3.25]) and $x \equiv 0$ satisfies both, the initial condition and the equation for Caputo derivative. Then one has $x\left(T_{1}^{-}\right)=0, \dot{x}_{0}\left(T_{1}^{-}\right)=0$ as initial conditions, which implies the solution $x \equiv 0$ in the second interval. Recursively one has as solution $x \equiv 0$. A contraction map argument on the operator, defined in the space of the continuous functions, $\mathcal{T}(\xi)(t):=$ $\int_{T_{i}}^{s} \tau^{\alpha-1} E_{\alpha, \alpha}(A, \tau) Q(t-\tau) \xi(t-\tau) d \tau$ proves that the solution $x \equiv 0$ is unique 
ROBUSTNESS AND CONVERGENCE OF FRACTIONAL ... 907

in $\mathcal{C}\left[T_{i}, s\right)$ for all $i \in \mathbb{N}$ where $s$ is such that $\| \int_{T_{i}}^{s} \tau^{\alpha-1} E_{\alpha, \alpha}(A, \tau) Q(t-$ $\tau) d \tau \|<1$. Recursively, one can extend for the total interval $\mathcal{C}\left[T_{i}, T_{i+1}\right)$.

Suppose now that there exists $x \neq y$ in $\mathcal{C}[0, T)$ with $x(0)=y(0)$ such that $D^{\alpha} x=(A+Q(t)) x+\nu$ and $D^{\alpha} y=(A+Q(t)) y+\nu$. Therefore, $D^{\alpha}(x-y)=(A+Q(t))(x-y)$ with $(x-y)(0)=0$, which gives as solution $x-y \equiv 0$. We conclude that the assumption $x-y \neq 0$ is a contradiction and thus, the continuous solution is unique.

2.5. Mixed order systems. In the previous results, the same order of derivation was used for each component of vector function $x$ in its defining equation. We present now the generalization when different orders of derivation for each component of vector $x$ are allowed.

TheOREM 2.6. Let $D^{\alpha} x(t)$ be a vector of components $D^{\alpha_{i}} x_{i}(t)$ where $0<\alpha_{i}<2$ for $i=1, \ldots, n$ and $x(t) \in \mathbb{R}^{n}$ for all $t \geq 0$. Consider the Caputo system

$$
D^{\alpha} x(t)=(A+Q(t)) x(t)+f(x(t))+\nu(t),
$$

where $\nu:[0, \infty) \rightarrow \mathbb{R}^{n}$ is a bounded piece-wise continuous function and $Q$ : $[0, \infty) \rightarrow \mathbb{R}^{n \times n}$ is a piece-wise continuous with $\lim _{t \rightarrow \infty} Q(t)=0$. Suppose that $A, Q(t) \in \mathbb{R}^{n \times n}$ are triangular matrices (without loss of generality, lower triangular both) such that

$$
\sigma(A) \subset\left\{\lambda \in \mathbb{C} \backslash\{0\}:|\arg (\lambda)|>\frac{\alpha_{M} \pi}{2}\right\},
$$

where $\alpha_{M}=\max _{i}\left\{\alpha_{i}\right\}$. $f_{i}$ for any $i=1, \ldots, n$ are locally Lipschitz continuous with respect to $x_{i}$ around the origin, uniformly with respect to the other variables, with Lipschitz constant $L_{i}$ such that $\lim _{r \rightarrow 0^{+}} L_{i}(r)=0$ for the neighborhood around the origin $B(0, r)$ and such that $f(0)=0$.

(i) Suppose $f(x) \equiv 0$. Then, $x$ is a bounded continuous function and if $\nu$ converges to zero, $x$ converges to zero.

(ii) Suppose $\nu \equiv 0$ and $f_{i}=f_{i}\left(x_{1}, \ldots, x_{i}\right)$ for any $i=1, \ldots, n$. Then, $x$ converges to zero.

P r o o f. Since the diagonal of $A$ are its eigenvalues, a recursive application of Theorem 2.1 (Corollary 2.1) for the scalar equation in each $x_{i}$ for $i=1, \ldots, n$ yields the desired result for the (i) part and a recursive application of Theorem 2.6 yields the desired result for (ii) part.

REMARK 2.5. Theorem 2.7(i) is the extension of the BIBO stability result [13, Theorem 1] for non commensurate systems. From Theorem 2.7(ii) one can obtains the corresponding first Lyapunov theorem for mixed order systems, using similar arguments as those used in the proof of Theorem 2.4 . 


\section{Robustness and convergence of fractional adaptive schemes}

In [9], it was stressed the importance in optimal control and/or identification adaptive problems of the Fractional Error Model of Type I defined by the following system of equations

$$
\left\{\begin{array}{l}
e=\phi^{T} w+\nu \\
D^{\alpha} \phi=-\gamma e w=-\gamma w w^{T} \phi-\gamma w \nu
\end{array}\right.
$$

where $D^{\alpha} \phi$ is a vector of components $D^{\alpha_{i}} \phi_{i}$ with $\alpha_{i} \in(0,2)$ for $i=1, \ldots, n$, $e:[0, \infty) \rightarrow \mathbb{R}$ is a measure of the adaptation error, $w:[0, \infty) \rightarrow \mathbb{R}^{n}$ is the information signal, $\phi:[0, \infty) \rightarrow \mathbb{R}^{n}$ is the parameter error and $\nu:[0, \infty) \rightarrow \mathbb{R}$ is a bounded perturbation function due to noise, imperfect modeling and/or initial conditions terms. $\gamma>0$ is an scalar or matrix parameter to be suitably chosen to handle the speed of convergence. The parameter $\alpha$ turns out to be a relevant degree of freedom for optimization purposes $([9, \S 5])$. Without loss of generality, it is assumed that $w$ is bounded since it can be normalized by using $\gamma\left(\right.$ e.g. $\left.\gamma:=\frac{\gamma^{\prime}}{1+w^{T} w}\right)$. For simplicity, we will assume $\gamma=1$.

Since $w$ is bounded, the robust adaptation objective will be reached, namely $\lim _{t \rightarrow \infty} e=0$ when $\nu \equiv 0$ and $e$ bounded if $\nu$ is bounded, if conditions on $w$ are such that $\phi$ converges to zero or remains bounded, respectively. The results of Section 2 (Theorem 2.1 or Theorem 2.2 in particular) imply this if we are able to express $w$ as

$$
-w(t) w^{T}(t) \leq A+Q(t)
$$

with $Q$ satisfying (2.4) (or $\sup _{t>T}\|Q(s)\|$ small enough) and a suitable matrix $A$. The set of $w$ is allowed to be piece-wise continuous by Theorem 2.5 (cf. [9, Theorem 2, Lemma 3]) where differentiability on $w$ was required when $\nu \equiv 0)$. By applying Theorem 2.3 and Remark 2.4, we can extend the fractional adjustment to $\alpha \geq 1$ whereas in [9] it was proved only for $\alpha \in(0,1]$ when $\nu \equiv 0$. We remark that since the choice of the order of derivation on the adjustment parameters law is a relevant variable of optimization [9, Example 5], the established theorems effectively ample the tools for the designer of adaptive schemes.

3.1. Ideal case: $\nu \equiv 0$. Note first that if $A$ is chosen with negative real eigenvalues (e.g. $A=-\epsilon I$ for $\epsilon>0$ ), then $\sigma(A) \subset \Lambda_{\alpha}^{s}$ for any $\alpha \in(0,2)$.

For the scalar case, condition (3.2) takes the form $w^{2} \geq a+q(t)$ where $a>0$. If $w^{2}=\epsilon$ then we choose $a=\epsilon$ and $q=0$. If $\lim _{t \rightarrow \infty} w^{2}(t)=\epsilon$ we choose $a=\epsilon$ and $q=w^{2}-\epsilon$. If $w^{2}=\sin ^{2} t$, given that $\sin ^{2} t>\epsilon$ 'most of the time' if $\epsilon>0$ is small enough, we define $a=\epsilon$ and $q(t)$ zero everywhere but on small intervals where it takes the value $w^{2}-\epsilon$ whereby $|q|<\epsilon$ but a point of null measure ( $q$ piece wise continuous). 
ROBUSTNESS AND CONVERGENCE OF FRACTIONAL ... 909

The above examples are persistently exciting signals i.e. functions $w$ such that there exist $T_{0}>0$ and $\epsilon>0$ satisfying $\int_{t}^{t+T_{0}} w w^{T} d \tau \geq \epsilon T_{0} I$ for any $t \geq 0$ where $I$ is the identity matrix. It is known that for $\alpha=1$, these signals make to converge $\phi$ to zero (see e.g. [15, §1]). Note that any $0<\varepsilon<\epsilon$ and the same $T_{0}>0$ is a valid choice. It appears natural to try to write them as $w(t) w^{T}(t) \geq \epsilon I+Q(t)$ where $Q(t)$ vanishes in $\left[t, t+T_{0}\right]$ or $w(t) w^{T}(t)-\epsilon I$ (with norm less or equal than $\epsilon$ ). To satisfy condition (3.2), we require to choose a small enough $\epsilon>0$ such that $\epsilon C(\alpha,-\epsilon I)<1$, which must exist since $E_{\alpha, \alpha}(t, A)$ is polynomial in $A$ (see e.g. [16, Equation (1.56)]), $C(\alpha, 0)=0$ and $C(\alpha,-\epsilon I)<\infty$.

Indeed, if there exists a sufficiently small $\epsilon<1$ such that $C(\alpha,-\epsilon I)=$ $\sup _{t \geq 0} \int_{0}^{t}\left\|\tau^{\alpha-1} E_{\alpha, \alpha}\left(-\tau^{\alpha} \epsilon I\right)\right\| d \tau<1$ then any non negative almost periodic function $p=p(t)$ of amplitude $\epsilon$ is such that $\int_{0}^{t}\left\|\tau^{\alpha-1} E_{\alpha, \alpha}\left(-\tau^{\alpha} \epsilon I\right)\right\| p(t-$ $\tau) d \tau<1$ for every $t$. If for every $\epsilon>0, C(\alpha,-\epsilon I)>1$ then we can choose $\epsilon<1 / C(\alpha,-\epsilon I)$, whereby $\epsilon C(\alpha,-\epsilon I)<1$. Taking, $\|Q(t)\| \leq p(t)$, we have $Q$ holds condition (2.4). Hence, for any persistently exciting $w$, we have that $\phi$ and $e=\phi^{T} w$ converge to zero.

3.2. Perturbed case: $\nu \neq 0$. We first show that there exist bounded destabilizing perturbations justifying why it is worth to design robust schemes. Consider the scalar case and the following system

$$
\left\{\begin{array}{l}
e=\phi w+\nu \\
D^{\alpha} \phi=-e w \\
w=e \\
\nu=1
\end{array}\right.
$$

Consider $0<\alpha \leq 1$. Since $D^{\alpha} \phi(t)=-\frac{1}{(1-\phi)^{2}} \leq 0$ for all $t \geq 0$, either $\phi$ is bounded or diverges to $-\infty$. However if $a<\phi(t)<b$ for some $a, b \in \mathbb{R}$ then $c<D^{\alpha} \phi<d<0$ for negative numbers $c, d \in \mathbb{R}$. By $\alpha$-integrating $D^{\alpha} \phi<d$ we obtain a contradiction since $\phi$ converges and in particular it is bounded at infinity. Then $\phi$ diverges to $-\infty$ and $\lim _{t \rightarrow \infty} e=\lim _{t \rightarrow \infty} \frac{\nu}{1-\phi}=0$ (note that $w$ is bounded).

Therefore, $\nu \equiv 1$ is a bounded destabilizing perturbation when $w=e$. Note that $w$ is not persistently exciting since $\lim _{t \rightarrow \infty} w(t)=0$. A similar construction was done in [15, §III.2] but for the integer Error Model of Type II applied in adaptive control problem.

When $\alpha=1, w$ is persistently exciting and $\nu \equiv 0$, it was proved in [14] that $\phi$ converges exponentially to zero. Hence, the state transition matrix $\Phi\left(t, t_{0}\right)$ also converges exponentially to zero (recall that $\phi(t)=$ $\left.\Phi\left(t, t_{0}\right) \phi\left(t_{0}\right)\right)$ and the solution to (3.1) can be written as 


$$
\phi(t)=\Phi\left(t, t_{0}\right) \phi\left(t_{0}\right)-\int_{0}^{t} \Phi\left(t-\tau, t_{0}\right) \nu(\tau) w(\tau) d \tau
$$

where $\nu w$ is bounded since $\nu, w$ are bounded, the integral term of (3.3) is bounded, whereby $\phi$ is bounded. We postulate for the system

$$
D^{\alpha} x(t)=A(t) x(t),
$$

a matrix function $\Phi(t)$ so that $x(t)=\Phi(t) x(0)$ for any $x(0) \in \mathbb{R}^{n}$; as Caputo derivative is initialized at $t=0$, there is no need to use $\Phi\left(t, t_{0}\right)$ since $t_{0}=0$ is fixed. By $\alpha$-differentianting $x(t)=\Phi(t) x(0)$ and given that $x(0)$ is arbitrary, function $\Phi(t)$ must satisfies $D^{\alpha} \Phi(t)=A(t) \Phi(t)$ with $\Phi(0)=I$, the identity matrix. With this matrix one postulates as solution to the system

$$
D^{\alpha} x(t)=A(t) x(t)+f(x(t), t),
$$

the expression (generalizing (3.3) for $\alpha<1$ )

$$
x(t)=\Phi(t) x(0)+I^{\alpha}[\Phi(t-\cdot) f(x(\cdot), \cdot)](t) .
$$

By taking Caputo $\alpha$-derivative of (3.4) and using that $D^{\alpha} I^{\alpha}[g(\cdot)](t)=$ $g(t)$ for any $g$ continuous function [6. Theorem 3.7], we obtain $D^{\alpha} x(t)=$ $A(t) x(t)+f(x, t)$. By uniqueness of the solution, we conclude that (3.4) is the solution. However, in the revised literature there is no condition to estimate the speed of convergence so to have $\Phi(t) \in \mathcal{L}^{1}$ (see Remark 2.1(ii)). In the proof of [10, Theorem 2] an estimate for the speed of convergence of $x$ can be deduced but it is not enough to conclude that $\Phi(t) \in \mathcal{L}^{1}$ and in [5. Lemma 3.1] non negative Lyapunov exponent were stated for linear time varying Caputo fractional systems.

If $w$ is persistently exciting or such that (3.2) holds. Then for bounded perturbation $\nu$ in the error measurement, we have that the solution to equation (3.1) remains bounded by applying Theorem 2.1 or Theorem 2.2 since $\nu w$ is a bounded function, which gives a robustness result for adaptive schemes without modifying the adaptive laws, and moreover, guaranteeing that for decaying to zero disturbances their properties remains unchanged, that is $\phi$ converges to zero.

3.3. Error Model of Type II. This model is defined by the system

$$
\left\{\begin{array}{l}
D^{\alpha} e=A e+\phi^{T} w+\nu, \\
D^{\beta} \phi=-e w
\end{array}\right.
$$

where $e, v:[0, \infty) \rightarrow \mathbb{R}^{n}, w, v:[0, \infty) \rightarrow \mathbb{R}^{m}$ and $\alpha, \beta$ are seen as vectors of non negative components. In [9], implicit conditions were established for the convergence to zero of $e$ when $\alpha=\beta \leq 1$. Defining $x=\left(e^{T}, \phi^{T}\right)^{T}$, $\gamma=\left(\alpha^{T}, \beta^{T}\right)^{T}$, system (3.5) is equivalent to 
ROBUSTNESS AND CONVERGENCE OF FRACTIONAL ... 911

$$
D^{\gamma} x=\left[\begin{array}{cc}
A & w^{T} \\
-w & 0
\end{array}\right] x+\left[\begin{array}{l}
\nu \\
0
\end{array}\right]
$$

To apply the theorems of $\S 2$ guaranteeing convergence to zero of $(e, \phi)$ if $\nu \equiv 0$ and boundedness of $(e, \phi)$ if $\nu$ is bounded, we must write

$$
\left[\begin{array}{cc}
A & w^{T}(t) \\
-w(t) & 0
\end{array}\right] \geq \Lambda+Q(t)
$$

for suitable $\Lambda$ and $Q$. If, for example $w, e$ are scalars, $w=w_{0} \neq 0$ a constant and $A=-a<0$, then we can take $\Lambda:=\left[\begin{array}{cc}-a & w_{0} \\ -w_{0} & 0\end{array}\right]$ (its characteristic polynomial is $\left.\lambda^{2}+a \lambda+w_{0}^{2}\right)$ and $Q=0$. If $w=w_{0}+q(t)$ where $q$ converges to zero or it is small enough, we define $\Lambda+Q:=\left[\begin{array}{cc}-a & w_{0} \\ -w_{0} & 0\end{array}\right]+\left[\begin{array}{ll}0 & q \\ q & 0\end{array}\right]$. Note that $\|Q(t)\|_{F}=|q(t)|\left\|\left[\begin{array}{ll}0 & 1 \\ 1 & 0\end{array}\right]\right\|_{F}=2|q(t)|$ and condition $\sup _{t \geq T}\|Q(t)\|<$ $\frac{1}{C(\alpha, A)}$ means $\|q\|_{\infty}$ small enough.

When $\alpha=1, \nu \equiv 0$ and $w$ is such that for all $t>0$ and for all constant unit vector $u \in \mathbb{R}^{n}$ there exists $T_{0}, \epsilon$ with $\left|\int_{t}^{t+T_{0}} u^{T} w(\tau) d \tau\right| \geq \epsilon T_{0}$, the system is exponentially stable, whereby if $\nu$ is bounded $x$ is bounded (see e.g. [15]). It seems natural to look into the set of functions $w$. Note that this condition implies that we can project $w(t)= \pm \epsilon u+q(t)$ where $q(t)$ is such that $\int_{t}^{t+T_{0}} u^{T} q(t) d \tau \geq 0$. Then, we can write $\Lambda+Q(t)=$ $\left[\begin{array}{cc}-a & \pm \epsilon u^{T} \\ \mp \epsilon u & -\mu I\end{array}\right]+\left[\begin{array}{cc}0 & q^{T} \\ -q & \mu I\end{array}\right]$, where $\mu>0$ is chosen small enough. The conditions for $Q$ are similar as in $\S 3.1$.

\section{Acknowledgements}

The authors thank CONICYT-Chile for the support under Grant No FB0809 'Centro de Tecnologia para la Mineria' and FONDECYT 1150488 'Fractional Error Models in Adaptive Control and Applications'.

\section{References}

[1] N.D. Cong, T.S. Doan, H.T. Tuan, Asymptotic stability of linear fractional systems with constant coefficients and small time dependent perturbations. arXiv:1601.06538v1 [math.DS], January 2016.

[2] N.D. Cong, T.S. Doan, S. Siegmund, and H.T. Tuan, Linearized asymptotic stability for fractional differential equations. Electron. J. Qual. Theory Differ. Equ. 39 (2016), 1-13. 
[3] N.D. Cong, T.S. Doan, H.T. Tuan, An instability theorem of nonlinear fractional differential systems. arXiv:1603.07904v1 [math.DS], Apr. 2016.

[4] N.D. Cong, T.S. Doan, H.T. Tuan, A Perron-type theorem for fractional linear differential systems. arXiv:1602.04695v2 [math.CA], June 2016.

[5] N.D. Cong, T.S. Doan, H.T. Tuan, On fractional Lyapunov exponent for solutions of linear fractional differential equations, Fract. Calc. Appl. Anal. 17, No 2 (2014), 285-306; DOI: 10.2478/s13540-014-0169$1 ;$ https://www.degruyter.com/view/j/fca.2014.17.issue-2/ issue-files/fca.2014.17.issue-2.xml.

[6] K. Diethelm. The Analysis of Fractional Differential Equations. An Application-Oriented Exposition Using Differential Operators of $\mathrm{Ca}$ puto Type. Lecture Notes in Mathematics \# 2004, Springer-Verlag, Berlin, 2010.

[7] M.A. Duarte-Mermoud, N. Aguila-Camacho, J.A. Gallegos, R. CastroLinares, Using general quadratic Lyapunov functions to prove Lyapunov uniform stability for fractional order systems. Commun. Nonlinear Sci. Numer. Simul. 22, No 1 (2014), 650-659.

[8] J. A. Gallegos, M. Duarte-Mermoud. On Lyapunov theory for fractional system. Applied Mathematics and Computation 287 (2016), 161-170.

[9] J.A. Gallegos, M. Duarte-Mermoud, Fractional adaptivity using gradient method. ISA Trans. 69 (2017), 31-42; DOI: 10.1016/j.isatra.2017.04.021.

[10] J.A. Gallegos, M.A. Duarte-Mermoud, Boundedness and convergence on fractional order systems. J. of Computational and Applied Mathematics 296, Issue $\mathrm{C}$ (2016), 815-826.

[11] J.A. Gallegos, M.A. Duarte-Mermoud, On fractional extensions of Barbalat lemma. System and Control Letters 84, No 1 (2015), 7-12.

[12] A.A. Kilbas, H.M. Srivastava and J.J. Trujillo. Theory and Applications of Fractional Differential Equations. North-Holland Mathematics Studies \# 204, Elsevier Science B.V., Amsterdam, 2006.

[13] R. Malti, A note on $p$-norms of fractional systems. Automatica (J. of IFAC) 49, No 9 (2013), 2923-2927.

[14] A. Morgan and K. Narendra, On the uniform asymptotic stability of certain nonautonomous linear differential equations. SIAM J. Control

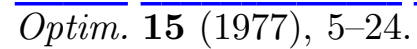

[15] K. Narendra, A. Annaswamy, Robust adaptive control in presence of bounded disturbance. IEEE Trans. Automat. Control 31 (1985), 306315.

[16] I. Podlubny, Fractional Differential Equations. Academic Press, San Diego, 1999. 
ROBUSTNESS AND CONVERGENCE OF FRACTIONAL . . 913

[17] C. Sin, L. Zheng, Existence and uniqueness of global solutions of caputo-type fractional differential equations. Fract. Calc. Appl. Anal. 19, No 3 (2016), 765-774; DOI: 10.1515/fca-2016-0040; https://www.degruyter.com/view/j/fca.2016.19.issue-3/ issue-files/fca.2016.19.issue-3.xml.

[18] H.T. Tuan, A note about the linearized stability of a class of nonlinear fractional differential systems. arXiv:1608.07906v1 [math.DS] 29 Aug. 2016.

${ }^{1}$ Department of Electrical Engineering University of Chile

Av. Tupper 200\%, Santiago, CHILE

Received: September 15, 2016

e-mail: jgallego@ing.uchile.cl

Revised: June 1, 2017

${ }^{2}$ Department of Electrical Engineering

\& Advanced Mining Technology Center (AMTC)

University of Chile

Av. Tupper 200\%, Santiago, CHILE

e-mail:mduartem@ing.uchile.cl

Please cite to this paper as published in:

Fract. Calc. Appl. Anal., Vol. 20, No 4 (2017), pp. 895-913,

DOI: $10.1515 /$ fca-2017-0047 